University of Nebraska - Lincoln

DigitalCommons@University of Nebraska - Lincoln

$10-5-2009$

\title{
Reexamine structures and relative stability of medium-sized silicon clusters: Low-lying endohedral fullerene-like clusters $\mathrm{Si}_{30}-\mathrm{Si}_{38}$
}

Soohaeng Yoo

University of Nebraska-Lincoln

Nan Shao

University of Nebraska-Lincoln

Xiao Cheng Zeng

University of Nebraska-Lincoln, xzeng1@unl.edu

Follow this and additional works at: https://digitalcommons.unl.edu/chemzeng

Part of the Chemistry Commons

Yoo, Soohaeng; Shao, Nan; and Zeng, Xiao Cheng, "Reexamine structures and relative stability of mediumsized silicon clusters: Low-lying endohedral fullerene-like clusters $\mathrm{Si}_{30}-\mathrm{Si} 38$ " (2009). Xiao Cheng Zeng Publications. 97.

https://digitalcommons.unl.edu/chemzeng/97

This Article is brought to you for free and open access by the Published Research - Department of Chemistry at DigitalCommons@University of Nebraska - Lincoln. It has been accepted for inclusion in Xiao Cheng Zeng Publications by an authorized administrator of DigitalCommons@University of Nebraska - Lincoln. 


\title{
Reexamine structures and relative stability of medium-sized silicon clusters: Low-lying endohedral fullerene-like clusters $\mathrm{Si}_{30}-\mathrm{Si}_{38}$
}

\author{
Soohaeng Yoo, Nan Shao, and X. C. Zeng \\ Department of Chemistry and Nebraska Center for Materials and Nanoscience, \\ University of Nebraska-Lincoln, Lincoln, NE 68588, USA \\ Corresponding author - X. C. Zeng, email xzeng1@unl.edu
}

\begin{abstract}
We report improved results of lowest-lying silicon clusters $\mathrm{Si}_{30}-\mathrm{Si}_{38}$. A large population of low-energy clusters are collected from previous searches by several research groups and the binding energies of these clusters are computed using density-functional theory (DFT) methods. Best candidates (isomers with high binding energies) are identified from the screening calculations. Additional constrained search is then performed for the best candidates using the basin-hopping method combined with DFT geometry optimization. The obtained lowlying clusters are classified according to binding energies computed using either the Perdew-Burke-Ernzerhof (PBE) functional or the Becke exchange and Lee-Yang-Parr correlation (BLYP) functional. We propose to rank low-lying clusters according to the mean PBE/ BLYP binding energies in view that the PBE functional tends to give greater binding energies for more compact clusters whereas the BLYP functional tends to give greater binding energies for less compact clusters or clusters composed of small-sized magic-number clusters. Except for $\mathrm{Si}_{30}$, the new search confirms again that medium-size silicon clusters $\mathrm{Si}_{31}-\mathrm{Si}_{38}$ constructed with proper fullerene cage motifs are most promising to be the lowest-energy structures.
\end{abstract}

\section{Introduction}

Structures of medium-sized silicon clusters have been investigated by many researchers over the last five years [1-20] using various global optimization methods [21-26]. Numerous theoretical studies of medium-sized neutral clusters $\mathrm{Si}_{N}$ in the size range $N=25-40$ have shown that beyond $N=29$, compact and spherical-like structures are more stable than elongated structures $[4,8$, $11,12,15]$ and that carbon fullerene cages tend to be generic cage motifs for low-lying spherical-like clusters $[4,9,11-13,15-20]$. To date, a large population of low-energy clusters of $\mathrm{Si}_{N}(N=25-40)$ have been obtained from independent global-minimum searches by several research groups: (1) an unbiased search [4] using genetic algorithm [21] combined with the non-orthogonal tightbinding method [27]. (2) an unbiased search [11, 12] using minima-hopping method [25] combined with the density-functional based tight-binding (DFTB) model [28], and (3) various biased searches (based on a large number of pre-constructed endohedral fullerene structures) using a compression method [20] combined with a tight-binding model of silicon [29], or a relaxation method combined with quantum molecular dynamics simulation [16], or a basin-hopping method combined with density-functional theory (DFT) geometry optimization [4, 8, 26]. Although for each size there are many candidates to compete for the lowest-energy structure, the homologue carbon cage motifs (carbon fullerenes [30]) and the number of core atoms inside the cages for most low-ly- ing clusters are reasonably established [11, 13, 16, 20]. Still, a challenge is to determine exact structures of the lowest-energy clusters (global minima). The challenge is mainly due to two subtleties: (1) high-level ab initio computation is very demanding or impractical for medium-sized silicon clusters [31], and (2) the energy rankings based on DFT total-energy computation can be sensitive to the density functional selected, such as the Perdew-Burke-Ernzerhof (PBE) functional [32], or the Becke exchange and Lee-Yang-Parr correlation (BLYP) functional [33]. In general, the PBE functional tends to give greater binding energies for more compact clusters (clusters with smaller cages but more core-filling atoms) whereas the BLYP functional tends to give greater binding energies for less compact clusters or clusters consisting of small-sized magic-number clusters such $\mathrm{Si}_{6}, \mathrm{Si}_{7}$ and $\mathrm{Si}_{10}[12,13]$.

\section{Method and calculation}

In this Letter, we present a systematic study of low-lying clusters $\mathrm{Si}_{N}(N=30-38)$. First, we have collected a large number of low-energy clusters reported in the literature by various groups $[4,11$, $13,16,20]$. Binding energies of these low-energy isomers are computed using DFT methods within generalized-gradient approximation (GGA) implemented in the CPMD program [34]. For the binding-energy computation, we used a supercell length of $25 \AA$ and an energy cut-off of 30 Rydberg for plane-wave expansion. We adopted Troullier-Martins norm-conserving pseudopoten- 
Table 1. Optimal core/cage combination for low-lying endohedral silicon clusters $\mathrm{Si}_{30}-\mathrm{Si}_{40}$ reported in Ref. [16] (Ma et al.) and Ref. [20] (Zhou and Pan). The binding energies per atom are calculated at DFT level with two GGA functionals (PBE and BLYP). Isomers with the greatest PBE binding energy are highlighted in bold, and isomers with the greatest mean (PBE + BLYP) binding energy are highlighted with a frame. The si36-O' is obtained from structural perturbation of si36-O.

\begin{tabular}{|c|c|c|c|c|}
\hline & \multirow{2}{*}{$\begin{array}{l}\text { Optimal core/cage combination } \\
\text { (homologue carbon fullerene cage [30]) }\end{array}$} & \multicolumn{3}{|c|}{ Binding energy (eV/atom) } \\
\hline & & CPMD/PBE & CPMD/BLYP & Mean \\
\hline si30-O (Ref. [20]) & $\mathrm{Si}_{4} @ \mathrm{Si}_{26}\left[\mathrm{C}_{26}\left(D_{3:}: 1\right)\right]$ & 3.914 & 3.325 & 3.620 \\
\hline si30-Ma (Ref. [16]) & $\mathrm{Si}_{1} @ \mathrm{Si}_{2}\left[\mathrm{C}_{28}\left(D_{2}: 1\right)\right.$ & 3.886 & 3.322 & 3.604 \\
\hline si31-Ma & $\mathrm{Si}_{3} @ \mathrm{Si}_{28}\left[\mathrm{C}_{28}\left(T_{\mathrm{d}}: 2\right)\right]$ & 3.903 & 3.338 & 3.620 \\
\hline si32-O & $\mathrm{Si}_{4} @ \mathrm{Si}_{28}\left[\mathrm{C}_{28}\left(T_{\mathrm{d}}: 2\right)\right]$ & 3.916 & 3.340 & 3.628 \\
\hline si32-Ma & $\mathrm{Si}_{4} @ \mathrm{Si}_{28}\left[\mathrm{C}_{28}\left(T_{\mathrm{d}}: 2\right)\right]$ & 3.906 & 3.334 & 3.620 \\
\hline si34-O & $\mathrm{Si}_{6} @ \mathrm{Si}_{28}\left[\mathrm{C}_{28}\left(T_{\mathrm{d}}: 2\right)\right]$ & 3.932 & 3.330 & 3.631 \\
\hline si34-Ma & $\mathrm{Si}_{4} @ \mathrm{Si}_{30}\left[\mathrm{C}_{30}\left(\mathrm{C}_{2 \mathrm{v}}: 3\right)\right]$ & 3.906 & 3.346 & 3.626 \\
\hline si35-O & $\mathrm{Si}_{5} @ \mathrm{Si}_{30}\left[\mathrm{C}_{30}\left(C_{2}^{2 \mathrm{v}}: 2\right)\right]$ & 3.936 & 3.344 & 3.640 \\
\hline si35-Ma & $\mathrm{Si}_{3} @ \mathrm{Si}_{32}\left[\mathrm{C}_{32}\left(\mathrm{C}_{2}: 1\right)\right]$ & 3.914 & 3.351 & 3.632 \\
\hline si36-O & $\mathrm{Si}_{4} @ \mathrm{Si}_{32}\left[\mathrm{C}_{32}\left(D_{3}: 6\right)\right]$ & 3.936 & 3.356 & 3.646 \\
\hline si36-O' & $\mathrm{Si}_{4} @ \mathrm{Si}_{32}\left[\mathrm{C}_{32}\left(D_{3}: 6\right)\right]$ & 3.937 & 3.354 & 3.646 \\
\hline si36-Ma & $\mathrm{Si}_{4} @ \mathrm{Si}_{32}\left[\mathrm{C}_{32}\left(D_{3 \mathrm{~d}}: 3\right)\right]$ & 3.922 & 3.347 & 3.634 \\
\hline si39-Ma & $\mathrm{Si}_{5} @ \mathrm{Si}_{34}\left[\mathrm{C}_{34}\left(C_{\mathrm{s}}: 2\right)\right]$ & 3.941 & 3.362 & 3.651 \\
\hline si40-O & $\begin{array}{l}\mathrm{Si}_{6} @ \mathrm{Si}_{34} \\
\text { (having } 7 \text { member-ring) }\end{array}$ & 3.955 & 3.353 & 3.654 \\
\hline si40-Wang (Ref. [9]) & $\mathrm{Si}_{6} @ \mathrm{Si}_{34}\left[\mathrm{C}_{34}\left(C_{3 \mathrm{v}}: 6\right)\right]$ & 3.943 & 3.359 & 3.651 \\
\hline
\end{tabular}

tials [35] for PBE and Goedecker-Teter-Hutter (dual space Gaussian) norm-conserving pseudopotentials [36] for BLYP calculations. Top candidates (isomers with large binding energies) are identified from this screening calculation. Next, additional constrained searches are performed by using the basin-hopping (BH) method combined with DFT geometry optimization $[19,26]$. The obtained lowest-energy clusters are classified into three groups: one according to the binding energies computed based on the PBE functional, the second according to the binding energies based on the BLYP functional, and the third according to the mean PBE/BLYP binding energies.

\section{Results and discussion}

Table 1 shows calculated binding energies of low-lying endohedral clusters $\mathrm{Si}_{N}(N=30-40)$, reported by Ma et al. [16] and Pan and Zhou [20], respectively, as well as their corresponding homologue carbon cages labeled in the Fowler-Manolopoulos fullerene notation (including the point-group symmetry and the FowlerManolopoulos label [30]). These endohedral fullerene-like low-lying clusters were pre-constructed based on an empirical rule [4], that is, the $\mathrm{Si}_{m+3} / \mathrm{Si}_{m}(m=1,2, \ldots)$ is an upper/lower limit for the number of core filling atoms to be enclosed in a $\mathrm{Si}_{26+2 m}$ fullerene cage. Pan and Zhou employed a compression method combined with a tight-binding model of silicon, while Ma et al. relaxed the clusters using a quantum molecular dynamics method. The compression method seems more effective to generate compact low-lying clusters. The most notable results are the predicted homologue carbon fullerene cage $\mathrm{C}_{28}$ for $\mathrm{Si}_{32}$ and $\mathrm{Si}_{33}$ (a larger $\mathrm{C}_{30}$ cage was reported in Ref. [13]) and $C_{32}$ cage for $\mathrm{Si}_{38}$ (a larger $\mathrm{C}_{34}$ cage was reported in Ref. [13]). With these new cage motifs, our additional $\mathrm{BH}-\mathrm{DFT} / \mathrm{PBE}$ search yields new leading candidates for the lowestenergy clusters of $\mathrm{Si}_{32}$ and $\mathrm{Si}_{38}$, respectively, namely, si32-1a and si38-1a (see Table 2). We also confirm structure of the lowest-energy cluster $\mathrm{Si}_{33}$, namely, si33-1a. Pan and Zhou reported new lowlying structures of $\mathrm{Si}_{30}, \mathrm{Si}_{31}, \mathrm{Si}_{35}$ and $\mathrm{Si}_{36}$ with slightly improved DFT/PBE binding energies (typically $4-8 \mathrm{meV} /$ atom, which are comparable to typical error bar in DFT binding-energy computation $\sim 5 \mathrm{meV} /$ atom). The corresponding homologue carbon fullerene cages $\left(C_{28}\right.$ for $\mathrm{Si}_{30}$ and $\mathrm{Si}_{31}, \mathrm{C}_{30}$ for $\mathrm{Si}_{35}$, and $\mathrm{C}_{32}$ for $\left.\mathrm{Si}_{36}\right)$ are the same in size as reported Refs. 12 and 13. Our additional BH-DFT/ PBE search confirms these leading candidates. We thus name them as new si30-1a, si31-1a, si35-1a and si36-1a, respectively (see Table 2). We also predict a new leading candidate for the lowest-energy cluster $\mathrm{Si}_{37}$, named as si37-1a, whose binding energy is improved by $9 \mathrm{meV} /$ atom (see Table 2 and Reference [13]).

Table 2 also shows new results of low-lying endohedral fullerene-like clusters $\mathrm{Si}_{31}, \mathrm{Si}_{32}, \mathrm{Si}_{35}, \mathrm{Si}_{36}, \mathrm{Si}_{38}$ and $\mathrm{Si}_{39}$ based on DFT/ BLYP binding-energy computation, namely, si31-1a', si32-1a', si35-1a', si36-1a', si38-1a', and si39-1a', respectively. In particular, the bind energies of si32-1a' and si36-1a' are improved by 8 and $10 \mathrm{meV} /$ atom compared to those reported in Ref. [13]. At DFT/ BLYP level of theory, the Y-shaped three-arm clusters still give rise to the greatest binding energies for $\mathrm{Si}_{30}, \mathrm{Si}_{32}, \mathrm{Si}_{34}$ and $\mathrm{Si}_{37}$. Except for $\mathrm{Si}_{30}$, however, the binding-energy differences between the Y-shaped three-arm clusters (Ref. [13]) and the endohedral fullerene-like isomers (si32-1a', si34-1a' and si37-1a') are less than 6 $\mathrm{meV} /$ atom, comparable to the error bar $(\sim 5 \mathrm{meV} /$ atom $)$ in DFT energy computation.

In view of the dependence of energy rankings on the functional (e.g., PBE or BLYP) selected, we propose to use the mean $(\mathrm{PBE}+\mathrm{BLYP})$ binding energies to rank low-lying silicon clusters. As indicated above, the PBE functional tends to give greater binding energies for more compact clusters whereas the BLYP functional tends to give greater binding energies for less compact clusters or clusters consisting of small-sized magic-number clusters such $\mathrm{Si}_{6}, \mathrm{Si}_{7}$ and $\mathrm{Si}_{10}$. Hence, to some extent, the mean (PBE + BLYP) binding energy, when used as a measure of relative stability, balances the over preference of compact structure in the PBE calculation and the over preference of less compact structure in the BLYP calculation. The predicted low-lying clusters are also shown in Table 2, where the clusters with the greatest mean (PBE + BLYP) binding energies are highlighted by a frame and plotted in Figure 1(a). For references, the binding-en- 
Table 2. Optimal core/cage combination for leading candidates for the lowest-energy clusters $\mathrm{Si}_{30}-\mathrm{Si}_{40}$ and their binding energies per atom calculated at DFT level with two GGA functionals. "1a" denotes the lowest-lying isomer in PBE energy ranking (in bold), "1a"” denotes the lowest-lying isomer in BLYP energy ranking (in bold). " $1 \mathrm{~m}^{\prime}$ " denotes the isomer with the greatest mean (PBE + BLYP) binding energy. Also, isomers with the greatest mean binding energies are highlighted by a frame.

\begin{tabular}{|c|c|c|c|c|}
\hline & \multirow{2}{*}{$\begin{array}{l}\text { Optimal core/cage combination } \\
\text { (homologue fullerene cage [30]) }\end{array}$} & \multicolumn{3}{|c|}{ Binding energy (eV/atom) } \\
\hline & & CPMD/PBE & CPMD/BLYP & Mean \\
\hline $\mathrm{si30-1a}=\mathrm{si30-O}$ & $\mathrm{Si}_{4} @ \mathrm{Si}_{26}\left[\mathrm{C}_{26}\left(D_{3 \mathrm{~h}}: 1\right)\right]$ & 3.914 & 3.325 & 3.620 \\
\hline si30-1a' (Ref. [12]) & Y-Shape & 3.895 & 3.359 & 3.627 \\
\hline si31-1a' & $\mathrm{Si}_{3} @ \mathrm{Si}_{28}\left[\mathrm{C}_{28}\left(T_{\mathrm{d}}: 2\right)\right]$ & 3.891 & 3.347 & 3.619 \\
\hline si32-1a & $\mathrm{Si}_{4} @ \mathrm{Si}_{28}\left[\mathrm{C}_{28}\left(T_{\mathrm{d}}: 2\right)\right]$ & 3.926 & 3.342 & \begin{tabular}{|l|}
3.634 \\
\end{tabular} \\
\hline si32-1a' & $\mathrm{Si}_{4} @ \mathrm{Si}_{28}\left[\mathrm{C}_{28}\left(T_{\mathrm{d}}: 2\right)\right]$ & 3.919 & 3.347 & 3.633 \\
\hline si33-1a = si33-O & $\mathrm{Si}_{5} @ \mathrm{Si}_{28}\left[\mathrm{C}_{28}\left(T_{\mathrm{d}}: 2\right)\right]$ & 3.940 & 3.340 & 3.640 \\
\hline si35-1a & $\mathrm{Si}_{5} @ \mathrm{Si}_{30}\left[\mathrm{C}_{30}\left(C_{2 \mathrm{v}}: 3\right)\right]$ & 3.936 & 3.347 & 3.641 \\
\hline si35-1a' & $\mathrm{Si}_{3} @ \mathrm{Si}_{32}\left[\mathrm{C}_{32}\left(D_{3}: 6\right)\right]$ & 3.907 & 3.358 & 3.632 \\
\hline si36-1a & $\mathrm{Si}_{4} @ \mathrm{Si}_{32}\left[\mathrm{C}_{32}\left(D_{3}: 6\right)\right]$ & 3.937 & 3.355 & 3.646 \\
\hline si36-1a' & $\mathrm{Si}_{4} @ \mathrm{Si}_{32}\left[\mathrm{C}_{32}\left(D_{3 \mathrm{~d}}: 3\right)\right]$ & 3.934 & 3.364 & \begin{tabular}{|l|}
3.649 \\
\end{tabular} \\
\hline si37-1a & $\mathrm{Si}_{5} @ \mathrm{Si}_{32}\left[\mathrm{C}_{32}\left(D_{3}: 6\right)\right]$ & 3.949 & 3.348 & 3.649 \\
\hline si37-1a' (Ref. [13]) & $\mathrm{Si}_{5} @ \mathrm{Si}_{32}\left[\mathrm{C}_{32}\left(D_{3}: 6\right)\right]$ & 3.940 & 3.357 & 3.649 \\
\hline si37-1m = si37-O & $\mathrm{Si}_{5} @ \mathrm{Si}_{32}\left[\mathrm{C}_{3}\left(D_{3}: 6\right)\right]$ & 3.947 & 3.353 & 3.650 \\
\hline si39-1a' & $\mathrm{Si}_{5} @ \mathrm{Si}_{34}\left[\mathrm{C}_{34}\left(\mathrm{C}_{\mathrm{s}}: 2\right)\right]$ & 3.945 & 3.366 & 3.656 \\
\hline si40-1a (Ref. [19]) & $\mathrm{Si}_{6} @ \mathrm{Si}_{34}\left[\mathrm{C}_{34}\left(\mathrm{C}_{\mathrm{s}}: 3\right)\right]$ & 3.966 & 3.364 & 3.665 \\
\hline si40-1a' (Ref. [19]) & $\mathrm{Si}_{4} @ \mathrm{Si}_{36}\left[\mathrm{C}_{36}\left(D_{3 \mathrm{~h}}: 13\right)\right]$ & 3.928 & 3.372 & 3.650 \\
\hline
\end{tabular}

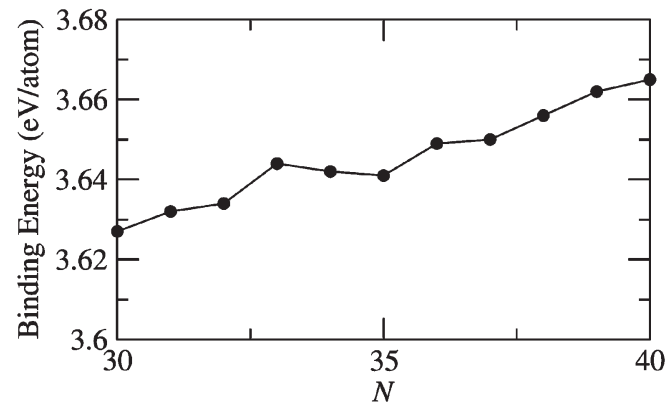

(a)

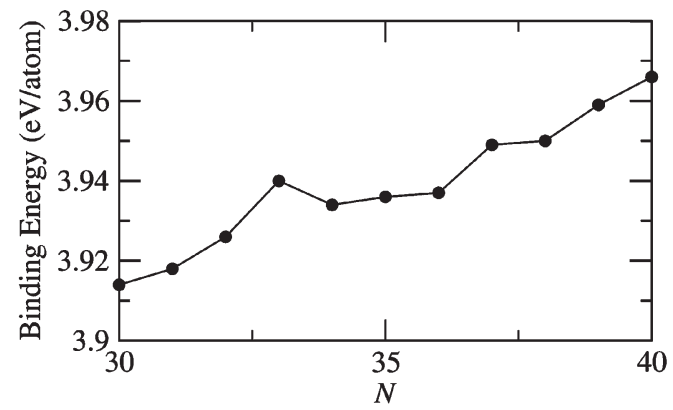

(b)

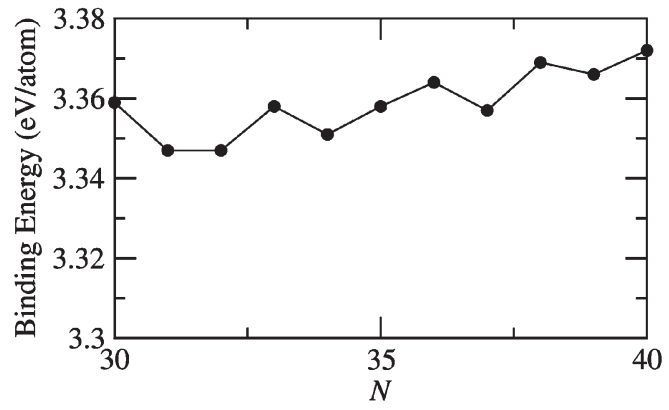

(c)

Figure 1. Binding energies per atom (eV/atom) of the predicted lowest-energy silicon fullerenes highlighted (in bold or with a frame) in Table 2: (a) mean (PBE + BLYP), (b) PBE, and (3) BLYP binding energies. A distinct peak can be seen at $N=33$ in (a) and (b).

ergies of the lowest-lying clusters $\mathrm{Si}_{39}$ and $\mathrm{Si}_{40}$ (see Ref. [19]) are also included in Table 2 and plotted in Figs. 1(a)-(c). Interestingly, only for $\mathrm{Si}_{30}$, the Y-shaped three-arm cluster (si30-1a') still has the greatest mean binding energy. This is likely because the cluster is composed of three highly stable magic-number subunits, i.e. two $\mathrm{Si}_{6}$ and one $\mathrm{Si}_{10}$ (Ref. [12]). For $\mathrm{Si}_{31}-\mathrm{Si}_{40}$, the endohedral fullerene-like clusters all give rise to the greatest mean binding energies. Specifically, for $\mathrm{Si}_{31}, \mathrm{Si}_{32}, \mathrm{Si}_{35}, \mathrm{Si}_{39}$ and $\mathrm{Si}_{40}$, the corresponding lowest-lying isomers predicted in DFT/PBE calculations also have the greatest mean binding energies, whereas for $\mathrm{Si}_{33}$ and $\mathrm{Si}_{36}$, the corresponding lowest-lying isomers predicted in DFT/BLYP calculation have the greatest mean binding energies. As shown in Figs. 1(a)-(c) and Table 2, the leading candidate for $\mathrm{Si}_{33}$, si33-1a', has greater mean binding energy than its two neighbour clusters. Thus, this isomer with $\mathrm{C}_{30}\left(C_{2 \mathrm{v}}: 3\right)$ homologue fullerene cage may be considered in future as a model cluster for the study of chemical reactivity of $\mathrm{Si}_{33}$ with small chemical molecules such as $\mathrm{C}_{2} \mathrm{H}_{2}$ and $\mathrm{NO}_{2}$ [37].

For $\mathrm{Si}_{37}$ and $\mathrm{Si}_{38}$, newly named isomers si37-1m [20] and si381m [13] (see Table 2) neither have the greatest binding energy in 
DFT/PBE calculation nor in DFT/BLYP calculation, but both have the greatest mean $(\mathrm{PBE}+\mathrm{BLYP})$ binding energy. For $\mathrm{Si}_{37}$, all three leading candidates (si37-1a, si37-1a' and si37-1m) entail the same homologue carbon fullerene cage $\mathrm{C}_{32}\left(D_{3}: 6\right)$, while for $\mathrm{Si}_{38}$, the two leading candidates (si38-1a' and si38-1m) entail the homologus carbon fullerene cage $\mathrm{C}_{34}\left(\mathrm{C}_{3 \mathrm{v}}: 6\right)$ and $\mathrm{C}_{34}\left(\mathrm{C}_{2}: 5\right)$, respectively.

\section{Conclusion}

In conclusion, we have performed constrained basin-hopping search for lowest-lying endohedral silicon clusters $\mathrm{Si}_{30}-\mathrm{Si}_{38}$, with initial structures screened from a large population of low-energy clusters obtained from previous searches by several research groups using different searching methods. New leading candidates for the lowest-energy clusters are obtained. These candidate clusters are classified into three groups: one according to the binding energies computed based the PBE functional, the second according to the binding energies based on the BLYP functional, and the third according to the mean PBE/BLYP binding energies. This is because the PBE functional tends to give greater binding energies for more compact clusters whereas the BLYP functional tends to give greater binding energies for less compact clusters or clusters composed of small-sized magic-number clusters. Except for $\mathrm{Si}_{30}$, the new $\mathrm{BH}$-DFT search confirms again that medium-size silicon clusters built with proper fullerene cage motifs are most promising to be the lowest-energy structures.

\section{Acknowledgments}

We are grateful to valuable discussions with Professors B. Pan and R. Zhou. This work is supported by grants from NSF (CHE0427746), the Nebraska Research Initiative, and by the Holland Computing Center at University of Nebraska.

\section{References}

[1] A. Sieck, Th. Frauenheim, and K. A. Jackson, Phys. Status Solidi B 240 (2003), p. 537.

[2] S. Yoo and X. C. Zeng, J. Chem. Phys. 119 (2003), p. 1442.

[3] S. Yoo, X. C. Zeng, X. Zhu, and J. Bai, J. Am. Chem. Soc. 125 (2003), p. 13318.

[4] S. Yoo, J. J. Zhao, J. L. Wang, and X. C. Zeng, J. Am. Chem. Soc. 126 (2004), p. 13845.

[5] N. N. Nair, T. Bredow, and K. Jug, J. Comput. Chem. 25 (2004), p. 1255.

[6] S. Ma, G. Wang, J. Wang, and L. Xue, Phys. Lett. A 337 (2005), p. 216.

[7] J. Zhao, J. Wang, J. Jellinek, S. Yoo, and X. C. Zeng, Eur. Phys. J. D 34 (2005), p. 35.

[8] S. Yoo and X. C. Zeng, J. Chem. Phys. 123 (2005), p. 164303.

[9] J. Wang, X. Zhou, G. Wang, and J. Zhao, Phys. Rev. B. 71 (2005), p. 113412.
[11] J. Bai, L. -F. Cui, J. Wang, S. Yoo, X. Li, J. Jellinek, C. Koehler, T. Frauenheim, L. -S. Wang, and X. C. Zeng, J. Phys. Chem. A 110 (2006), p. 908.

[12] S. Yoo and X. C. Zeng, J. Chem. Phys. 124 (2006), p. 054304.

[13] S. Yoo, N. Shao, C. Koehler, Th. Frauenheim, and X. C. Zeng, J. Chem. Phys. 124 (2006), p. 164311.

[14] R. L. Zhou and B. C. Pan, Phys. Rev. B 73 (2006), p. 045417.

[15] O. Ona, V. E. Bazterra, M. C. Caputo, J. C. Facelli, P. Fuentealba, and M. B. Ferraro, Phys. Rev. A 73 (2006), p. 053203.

[16] L. Ma, J. Zhao, J. Wang, B. Wang, and G. Wang, Phys. Rev. A 73 (2006), p. 063203.

[17] R. L. Zhou and B. C. Pan, Phys. Lett. A 368 (2007), p. 396.

[18] J. Wang, J. Zhao, L. Ma, and G. Wang, Eur. Phys. J. D 45 (2007), p. 289.

[19] S. Yoo, N. Shao, and X. C. Zeng, J. Chem. Phys. 128 (2008), p. 104316. F

[20] R. L. Zhou and B. C. Pan, Eur. Phys. J. D 47 (2008), p. 367.

[21] K. -M. Ho, A. A. Shvartsburg, B. Pan, Z. -Y. Lu, C. -Z. Wang, J. G. Wacker, J. L. Fye, and M. F. Jarrold, Nature 392 (1998), p. 582.

[22] D. J. Wales and H. A. Scheraga, Science 285 (1999), p. 1368.

[23] I. Rata, A. A. Shvartsburg, M. Horoi, Th. Frauenheim, K. W. M. Siu, and K. A. Jackson, Phys. Rev. Lett. 85 (2000), p. 546.

[24] K. A. Jackson, M. Horoi, I. Chaudhuri, T. Frauenheim, and A. A. Shvartsburg, Phys. Rev. Lett. 93 (2004), p. 013401.

[25] S. Goedecker, J. Chem. Phys. 120 (2004), p. 9911.

[26] S. Yoo and X. C. Zeng, Angew. Chem. Int. Ed. 44 (2005), p. 1491.

[27] M. Menon and K. R. Subbaswamy, Phys. Rev. B 50 (1994), p. 11577.

[28] D. Porezag, Th. Frauenheim, Th. Köhler, G. Seifert, and R. Kaschner, Phys. Rev. B 51 (1995), p. 12947.

[29] C. Z. Wang, B. C. Pan, and K. M. Ho, J. Phys. : Condens. Matter 11 (1999), p. 2043

[30] P. W. Fowler and E. D. Manolopoulos, An Atlas of Fullerenes, Clarendon, Oxford, UK (1995).

[31] X. Zhu, X. C. Zeng, Y. Lei, and B. Pan, J. Chem. Phys. 120 (2004), p. 8985.

[32] J. P. Perdew, K. Burke, and M. Ernzerhof, Phys. Rev. Lett. 77 (1996), p. 3865.

[33] A. D. Becke, Phys. Rev. A 38 (1988), p. 3098; J. P. Perdew, Phys. Rev. B 33 (1986), p. 8822; C. Lee, W. Yang, and R. G. Parr, Phys. Rev. B 37 (1988), p. 785;

[34] J. Hutter, A. Alavi, T. Deutsch, M. Bernasconi, S. Goedecker, D. Marx, M. Tuckerman, and M. Parrinello, CPMD, VERSION 3. 7. 1, MPI für Festkörperforschung Stuttgart, 2001.

[35] N. Troullier and J. L. Martins, Phys. Rev. B 43 (1991), p. 1993.

[36] S. Goedecker, M. Teter, and J. Hutter, Phys. Rev. B 54 (1996), p. 1703; C. Hartwigsen, S. Goedecker, and J. Hutter, Phys. Rev. B 58 (1998), p. 3641.

[37] J. L. Elkind, J. M. Alford, F. D. Weiss, R. T. Laaksonen, and R. E. Smalley, J. Chem. Phys. 87 (1987), p. 2397; S. Maruyama, L. R. Anderson, and R. E. Smalley, J. Chem. Phys. 93 (1990), p. 5349. 